\title{
ON FELLER'S KERNEL AND THE DRICHLET NORM
}

\author{
MASATOSHI FUKUSHIMA
}

\section{§ 0. Introduction}

Recently J. L. Doob [2] evaluated the Dirichlet integral of the BLD harmonic funtion on a Green space in terms of its fine boundary values and $\theta$-kernel of L. Naïm.

On the other hand, the general theory of additive functionals of Markov processes enables us to define the concept of the Dirichlet norm of functions with respect to Markov processes.

In $\S 1$ we shall prove that Naïm's kernel is equal to the kernel $U$, a generalization of the kernel introduced by W. Feller [3], on the Martin exit boundary of a Green space.

In $\S 2$ we shall treat the case of a multidimensional diffusion process corresponding to a self-adjoint elliptic differential operator. In this case we shall define the Dirichlet norm of the harmonic function and represent it in terms of Feller's kernel $U$, under certain regularity conditions.

The kernel $U$ plays an essential role in the investigation of the boundary problems concerning Markov processes ([3], [8]).

The author wishes to express his thanks to Prof. N. Ikeda and Prof. T. Watanabe for their kind suggestions and encouragement.

\section{§1. Feller's kernel and Naïm's kernel}

Let $p(t, x, y)$ be the Brownian transition density on a Green space $\mathrm{R}$. For $x, y \in \mathrm{R}$, put $G_{\alpha}(x, y)=\int_{0}^{+\infty} e^{-\alpha t} p(t, x, y) d t(\alpha \geqq 0)$, and $G(x, y)=G_{0}(x, y)$.

Let $\mathrm{M}$ be the totality of the minimal points of the Martin boundary of $\mathrm{R}$, and put $\hat{\mathrm{R}}=\mathrm{R} \cup \mathrm{M}$. To each point $y \in \hat{\mathrm{R}}$, there corresponds the Martin $K$ function $K(x, y), x \in \mathrm{R}$, which will sometimes be written as $K_{y}(x)$. When $y \in \mathrm{R}$, $K(x, y)=\frac{G(x, y)}{G\left(x_{0}, y\right)}$, where $x_{0}$ is a fixed reference point of $\mathrm{R}$.

Received December 2, 1963. 
For $y \in \hat{\mathrm{R}}-\left\{x_{0}\right\}$, we denote by $\mathfrak{B}^{y}=\left\{\Omega, \mathbf{B}, P_{x}^{y}, x \in \mathrm{R}^{\prime}, X_{t}, t \geqq 0\right\}$ the conditional Brownian motion on $\mathrm{R}^{\prime}$ whose transition density is $p^{y}(t, x, z)=\frac{K_{y}(z)}{K_{y}(x)} p(t, x, z)$, $\left(t>0, x, z \in \mathrm{R}^{\prime}\right)$, where $\mathrm{R}^{\prime}=\mathrm{R}-\{y\}$.

We adopt the notation $X \sigma_{\infty-}(w)=\lim _{t \uparrow \sigma \infty} X_{t}(w)$ where $\sigma_{\infty}$ is the life time of the path $w$. It holds that $X_{\sigma_{\infty}}(w)=y$ with $P_{x}^{y}$-measure 1. ([1]). The following definition is due to Kunita and Watanabe [6].

Definition 1. We call $y \in \mathrm{M}$ an exit boundary point if and only if there exists at least one point $x$ of $\mathrm{R}$ such that $P_{x}^{y}\left(\sigma_{\infty}<+\infty\right)>0$. If $P_{x}^{y}\left(\sigma_{\infty}<\infty\right)=0$ for every point $x$ of $\mathrm{R}$, then we call $y \in \mathrm{M}$ a passive boundary point.

The totality of the exit boundary points will be denoted by $(M)_{e x}$. Put $\hat{\mathrm{R}}_{1}=\mathrm{R} \cap(\mathrm{M})_{\text {ex }}$. Then we can check that $P_{x}^{y}\left(\sigma_{\infty}<+\infty\right)=1$ holds for $y \in \hat{\mathrm{R}}_{1}$ $-\left\{x_{0}\right\}, x \in \mathrm{R}^{\prime}$.

Now put

$K_{\alpha}(x, y)=K(x, y) E_{x}^{y}\left(e^{-\alpha \pi_{\infty}}\right)$, for $y \in \hat{\mathrm{R}}-\left\{x_{0}\right\}, x \in \mathrm{R}^{\prime}$, and $\alpha>0$.

Lemma 1.

i) For any $y \in \hat{\mathrm{R}}-\left\{x_{0}\right\}, \alpha>0$, the following equation holds.

$$
K(x, y)=\alpha \int_{\mathbf{R}} G_{\alpha}(x, z) K(z, y) d z+K_{\alpha}(x, y) \quad \text { for } x \in \mathrm{R}^{\prime} .
$$

ii) For any $y \in \hat{\mathrm{R}}_{1}-\left\{x_{0}\right\}, \alpha>0$, it holds that

$$
\begin{gathered}
K(x, y)=\alpha \int_{\mathrm{R}} G(x, z) K_{\alpha}(z, y) d z+K_{\alpha}(x, y) \quad \text { for } x \in \mathrm{R}^{\prime} . \\
\lim _{\alpha \rightarrow+\infty} K_{\alpha}(x, y)=0 \quad \text { for } x \in \mathrm{R}^{\prime} .
\end{gathered}
$$

iii) If $y$ in $\mathrm{M}$ is a passive boundary point,

$$
K_{\alpha}(x, y)=0 \quad \text { for any } \alpha>0, x \in \mathrm{R} .
$$

Proof. We only sketch the proof of ii). If $y \in \mathrm{R}_{1}-\left\{x_{0}\right\}$

$$
\begin{aligned}
\frac{\alpha}{K(x, y)} \int_{\mathrm{R}} G(x, z) K_{\alpha}(z, y) d z & =\alpha \int_{\mathrm{R}} \frac{K_{y}(z)}{K_{y}(x)} G(x, z) \frac{K_{\alpha}(z, y)}{K(z, y)} d z \\
& =\alpha E_{x}^{y}\left(\int_{0}^{\sigma_{\infty}} E_{x_{t}}^{y}\left(\epsilon^{-\alpha \sigma_{\infty}}\right) d t\right) .
\end{aligned}
$$

where $E_{x}^{y}$ denotes the expectation with respect to $P_{x}^{y}$-measure. According to the Markov property of $\mathfrak{B}^{y}$ and the fact that $\sigma_{\infty}$ is a Markov time, we find 
that the last term is equal to

$$
\alpha E_{x}^{y}\left(\int_{0}^{\sigma_{\infty}} e^{-\alpha\left(\sigma_{\infty}-t\right)} d t\right)=P_{x}^{y}\left(\sigma_{\infty}<+\infty\right)-E_{x}^{y}\left(e^{-\alpha \sigma_{\infty}}\right)=1-\frac{K_{\alpha}(x, y)}{K(x, y)} .
$$

Thus the equation (2) holds. Further we have

$$
\lim _{\alpha \rightarrow+\infty} \frac{K_{\alpha}(x, y)}{K(x, y)}=\lim _{\alpha \rightarrow+\infty} E_{x}^{y}\left(e^{-\alpha \sigma_{\infty}}\right)=P_{x}^{y}\left(\sigma_{\infty}=0\right)=0
$$

for any $x$ in $\mathrm{R}^{\prime}$.

From (1) and the resolvent equation, it follows that $K_{\alpha}(x, y)=\frac{G_{\alpha}(x, y)}{G\left(x_{0}, y\right)}$ for $y \in \mathrm{R}-\left\{x_{0}\right\}, x \in \mathrm{R}^{\prime}$.

Definition 2. For $x, y \in \hat{\mathrm{R}}-\left\{x_{0}\right\}$ and $\alpha>0, U_{\alpha}(x, y)$ is defined by

$$
U_{\alpha}(x, y)=\alpha \int_{\mathrm{R}} K(z, x) K_{\alpha}(z, y) d z .
$$

By the next lemma, we find that $U_{\alpha}$ is non-decreasing in $\alpha$. We put $U(x, y)=$ $\lim _{\alpha \rightarrow+\infty} U_{\alpha}(x, y)$, and we shall call it Feller's kernel with respect to the Brownian motion on $\mathrm{R}$.

$U_{\alpha}(x, y)$ has the meaning even if $x$ is a non-minimal boundary point. Therefore we can regard it as a $K$-potential as a function of $x$ in the sense of Naïm.

\section{Lemma 2.}

i) $U_{\alpha}(x, y)$ is not identically infinity, and

$$
\text { if } \alpha<\beta \text {, then } U_{\alpha}(x, y) \leqq U_{\beta}(x, y) \quad \text { for } x, y \in \hat{\mathrm{R}}-\left\{x_{0}\right\} \text {. }
$$

ii) If $x, y \in \hat{\mathrm{R}}_{1}-\left\{x_{0}\right\}$,

$$
U_{\alpha}(x, y)=\alpha \int_{\mathrm{R}} K_{\alpha}(z, x) K(z, y) d z=U_{\alpha}(y, x) .
$$

Proof.

i) When $y$ is the passive boundary point, $U_{\alpha}$ is zero. If $y \in \hat{\mathrm{R}}_{1}-\left\{x_{0}\right\}$, by (2), we can see that for $x \in \mathrm{R}^{\prime}-\left\{x_{0}\right\}$

$$
U_{\alpha}(x, y)=\alpha \int_{\mathrm{R}} \frac{G(z, x)}{G\left(x_{0}, x\right)} K_{\alpha}(z, y) d z:=\frac{K(x, y)-K_{\alpha}(x, y)}{G\left(x, x_{0}\right)}<+\infty .
$$

Further this equation shows that the inequality (5) holds for $x \in \mathrm{R}^{\prime}-\left\{x_{0}\right\}$, therefore according to one of the principal properties of the $K$-potential ([7] p. 200), (5) is true for any $x \in \hat{\mathrm{R}}-\left\{x_{0}\right\}$. 
ii) For $x \in \hat{\mathrm{R}}_{1}-\left\{x_{0}\right\}$ and the compact subset $\mathrm{D}$ of $\mathrm{R}$, let $K_{x}^{\mathrm{R}-\mathrm{D}}(z)$ be the extrémale of $K_{x}(z)$ relative to $\mathrm{R}-\mathrm{D}([7]$ p. 192$) . \quad K_{x}^{\mathrm{R}-\mathrm{D}}(z)$ can be written in the potential form,

$$
\int_{\mathrm{R}} G\left(z, z^{\prime}\right) \mu_{x}^{\mathrm{K}-\mathrm{D}}\left(d z^{\prime}\right)
$$

where $\mu_{x}^{\mathbf{R}-\mathbf{D}}$ is a non-negative measure on $R$. We note that

$$
K_{x}^{\mathrm{R}-\mathrm{D}}(z) \uparrow K_{x}(z) \text { when } \mathrm{D} \uparrow \mathrm{R} \text {. Putting } K_{\alpha, x}^{\mathrm{R}-\mathrm{D}}(z)=\int_{\mathrm{R}} G_{\alpha}\left(z, z^{\prime}\right) \mu_{x}^{\mathrm{R}-\mathrm{D}}\left(d z^{\prime}\right),
$$

we find, by the resolvent equation and (1), that

$$
\lim _{\mathrm{D} \uparrow \mathrm{R}} K_{\alpha, x}^{\mathrm{R}-\mathrm{D}}(z)=K_{\alpha}(z, x) .
$$

On account of (1) and (2),

$$
\alpha \int_{\mathrm{R}} K_{x}^{\mathrm{R}-\mathrm{D}}(z) K_{\alpha}(z, y) d z=\alpha \int_{\mathrm{R}} K_{\alpha, x}^{\mathrm{R}-\mathrm{D}}(z) K(z, y) d z
$$

for any $y \in \hat{\mathrm{R}}_{1}-\left\{x_{0}\right\}$.

Applying Fatou's lemma,

$$
U_{\alpha}(x, y)=\alpha \int_{\mathrm{R}} K(z, x) K_{\alpha}(z, y) d z \geqq \alpha \int_{\mathrm{R}} K_{\alpha}(z, x) K(z, y) d z=U_{\alpha}(y, x) .
$$

Since $x$ and $y$ are arbitrary in $\hat{\mathrm{R}}_{1}-\left\{x_{0}\right\}$, we have

$$
U_{\alpha}(y, x)=U_{\alpha}(x, y)
$$

which was to be proved.

Now, for $x, y \in \hat{\mathrm{R}}-\left\{x_{0}\right\}$, let $\theta(x, y)$ be Naïm's kernel. By definition, it is equal to $\frac{2 K(x, y)}{q G\left(x_{0}, x\right)}$ when $x \in \mathrm{R}-\left\{x_{0}\right\}, y \in \hat{\mathrm{R}}-\left\{x_{0}\right\}$, and equal to

$$
\lim _{\mathrm{D} \rightarrow \mathrm{R}} \int_{\mathrm{R}} \theta(z, y) G\left(x_{0}, z\right) \mu_{x}^{\mathrm{R}-\mathrm{D}}(d z) \text { when } x, y \in \hat{\mathrm{R}}-\left\{x_{0}\right\} .^{1)}
$$

Obviously $\theta(x, y)$ is symmetric in $x, y$. We shall prove the following theorem.

Theorem 1. For any $x, y \in \hat{\mathrm{R}}_{1}-\left\{x_{0}\right\}$,

$$
U(x, y)=\frac{q}{2} \theta(x, y) .
$$

1) We denote by $q$ either $2 \pi$ (if $N=2$ ) or the product of $N-2$ and the unit ball bound. ary area (if $N>2$ ), where $N$ is the dimension of $\mathrm{R}$. 
Proof. Let $y$ be fixed arbitrarily in $\hat{\mathrm{R}}_{1}-\left\{x_{0}\right\}$. If $x \in \mathrm{R}-\left\{x_{0}\right\}$ on account of (3) and (7),

$$
U(x, y)=\lim _{\alpha \rightarrow+\infty} U_{\alpha}(x, y)=\frac{K(x, y)}{G\left(x_{0}, x\right)}=\frac{q}{2} \theta(x, y) .
$$

When $x \in(\mathrm{M})_{e x}$, according to the principal properties of $K$-potintial and $\theta$-kernel ([7] p. 214), we find that

$$
U_{\alpha}(x, y)=\lim _{\substack{x^{\prime} \rightarrow x \\ x^{\prime} \in \mathbb{N}}} \frac{K\left(x^{\prime}, y\right)-K_{\alpha}\left(x^{\prime}, y\right)}{G\left(x_{0}, x\right)} \leqq \frac{\lim }{x^{\prime} \in \mathbb{R}} \frac{K\left(x^{\prime}, y\right)}{G\left(x_{0}, x^{\prime}\right)}=\frac{q}{2} \theta(x, y)
$$

Thus we have

$$
U(x, y) \leqq \frac{q}{2} \theta(x, y) \quad \text { for } x \in(\mathrm{M})_{\mathrm{ex}} \text { and } y \in \hat{\mathrm{R}}_{1}-\left\{x_{0}\right\} .
$$

On the other hand,

$$
\begin{aligned}
& \int_{\mathrm{R}} U_{\alpha}(x, z) G\left(x_{0}, z\right) \mu_{y}^{\mathrm{R}-\mathrm{D}}(d z)=\alpha \int_{\mathrm{R}} K_{\alpha}(z, x) K_{y}^{\mathrm{R}-\mathrm{D}}(z) d z \\
\leqq & \alpha \int_{\mathrm{R}} K_{\alpha}(z, x) K(z, y) d z=U_{\alpha}(x, y) .
\end{aligned}
$$

Letting $\alpha$ tend to infinity, we find that

$$
\int_{\mathrm{R}} U(x, z) G\left(x_{0}, z\right) \mu_{y}^{\mathrm{R}-\mathrm{D}}(d z) \leqq U(x, y)
$$

But since $U(x, z)$ in the integrand of the left member is equal to $\frac{q}{2} \theta(z, x)$ by (6) and (9), letting the compact subset $D$ tend to $R$, we obtain the following inequality.

$$
\frac{q}{2} \theta(x, y) \leqq U(x, y) . \quad \text { for } x \in(\mathrm{M})_{\text {ex }} \text { and } y \in \hat{\mathrm{R}}_{1}-\left\{x_{0}\right\}
$$

Remark. Assume that $\mathrm{M}=(\mathrm{M})_{\mathrm{ex}}$ and that there is no non-minimal boundary point. According to Doob [2], the above theorem implies that, for any BLD harmonic function $u$ on $\mathrm{R}$, the Dirichlet integral $\mathrm{D}(u)$ of $u$ can be represented in the following form.

$$
\mathbf{D}(u)=\int_{\mathrm{M}} \int_{\mathrm{M}}\left(u^{\prime}(x)-u^{\prime}(y)\right)^{2} U(x, y) \mu(d x) \mu(d y),
$$

where $u^{\prime}$ is the find boundary value of $u$ and $\mu(E)$ is the harmonic measure of a subset $E$ of $\mathrm{M}$ relative to the reference point $x_{0}$. 


\section{§ 2. The Dirichlet norm in the case of $A$-diffusion}

Let $\mathrm{D}$ be a bounded domain of the $N$-dimensional Euclidean space, whose boundary $\partial \mathrm{D}$ is a $(N-1)$-dimensional hypersurface of class $\mathbf{C}^{3}$.

Consider the self-adjoint elliptic differential operator $A$ expressed in terms of the local coodinates as

$$
A u(x)=\frac{1}{\sqrt{a(x)}} \frac{\partial}{\partial x^{i}}\left(a^{i j}(x) \sqrt{a(x)} \frac{\partial u(x)}{\partial x^{j}}\right)
$$

where $a^{i j}(x)$ is the contravariant tensor on $\overline{\mathrm{D}}$ which is strictly positive definite on $\overline{\mathrm{D}}$, and $a(x)=\operatorname{det}\left(a^{i j}(x)\right)^{-1.2)} \quad$ We assume that $a^{i j}(x)$ is a function of class $\mathbf{C}^{3}$ on $\overline{\mathrm{D}}$.

Let $p(t, x, y)$ be the fundamental solution of the diffusion equation

$$
\frac{\partial u(t, x)}{\partial t}=A u(t, x) \quad \text { for } x \in \mathrm{D}
$$

with the boundary condition, $u(t, x)=0$ for $x \in \partial \mathrm{D}$.

Let $\left\{\Omega, \mathrm{B}, P_{x}, x \in \mathrm{D}, X_{t}, t \geqq 0\right\}$ be a continuous strong Markov process with the transition probability $p(t, x, y)$ dy and it will be called an absorbing barrier A-diffusion [4].

According to S. Ito [5] and Ikeda, Ueno, Tanaka and Sato [4], we can verify the following related facts to this diffusion, We first note that $p(t, x, y)$ is symmetric in $x, y$. Put $G_{\alpha}(x, y)=\int_{0}^{+\infty} e^{-\alpha t} p(t, x, y) d t,(\alpha \geqq 0)$ and $G(x, y)=$ $G_{0}(x, y)$, for $x, y \in \mathrm{D}$, and put $h_{\alpha}(x, \xi)=h_{\alpha}\left(\xi, x\left(=\frac{\partial}{\partial n_{\xi}} G_{\alpha}(x, \xi)(\alpha \geqq 0)\right.\right.$ and $h(x, \xi)$ $=h_{0}(x, \xi)$ for $x \in \mathrm{D}, \xi \in \partial \mathrm{D}$. Further we define $U_{\alpha}(\xi, \eta)=\alpha \int_{\mathrm{D}} h_{\alpha}(\xi, z) h(z, \eta) d z$ for $\xi, \eta \in \partial \mathrm{D}$. 'Then we have

$$
\begin{array}{ll}
h(x, \xi)=\alpha \int_{\mathrm{D}} G(x, z) h_{\alpha}(z, \xi) d z+h_{\alpha}(x, \xi) & \text { for } x \in \mathrm{D}, \xi \in \partial \mathrm{D} \text { and } \alpha>0, \\
U_{\alpha}(\xi, \eta)=\int_{0}^{+\infty}\left(1-e^{-\alpha t}\right) \frac{\partial^{2}}{\partial n_{\xi} \partial n_{\eta}} p\left(t, \xi, \gamma_{1}\right) d t \quad \text { for } \xi, \eta \in \partial \mathrm{D} \text { and } \alpha>0 .
\end{array}
$$

We call

2) We denote by $d x, d \xi$ and $\frac{\partial}{\partial n}$, the volume element in $\mathrm{D}$, surface element in $\partial \mathrm{D}$ and normal derivative to the boundary point $\xi$ respectively, each of which is determined by the fundamental tensor $a^{i j}(x)$. Here the summation $\operatorname{sign} \sum_{i, j}$ is omitted as usual. 


$$
U(\xi, \eta)=\lim _{\alpha \uparrow+\infty} U_{\alpha}(\xi, \eta)=\int_{0}^{+\infty} \frac{\partial^{2}}{\partial n_{\xi} \partial n_{\eta}} p(t, \xi, \eta) d t
$$

Feller's kernel for our diffusion.

For the continuous function $u$ on $\partial \mathrm{D}$, we define $H u$ by

$$
H u(x)=\int_{\partial \mathrm{D}} h(x, \xi) u(\xi) d \xi .
$$

We have $\lim _{t \uparrow \infty_{\infty}} H u\left(X_{t}\right)=u\left(X_{\infty_{\infty-}}\right)$ and $H u(x)=E_{x}\left(u\left(X_{\infty_{\infty-}-}\right)\right)$ for $x \in \mathrm{D}$, where $\sigma_{\infty}$ is the life time of the path and $X_{\sigma_{\infty-}}=\lim _{t \uparrow \sigma_{\infty}} X_{t} \in \partial \mathrm{D}$. In our case $P_{x}\left(\sigma_{\infty}<\infty\right)=1$ holds for any $x \in \mathrm{D}$.

Now let us show that $v(x)=E_{x}\left(\left(u\left(X_{\sigma_{\infty}-}\right)-H u\left(X_{0}\right)\right)^{2}\right)$ is a potential.

Put

$$
\begin{aligned}
S_{t} & =H u\left(X_{t}\right)-H u\left(X_{0}\right) & & 0 \leqq t<\sigma_{\infty} \\
& =u\left(X_{\sigma_{\infty}-}\right)-H u\left(X_{0}\right) & & t \geqq \sigma_{\infty}
\end{aligned}
$$

Since $S_{t}$ is an additive functional and $E_{x}\left(S_{+\infty}\right)=0$, we obtain the equality,

$$
v(x)=E_{x}\left(S_{+\infty}^{2} ; t \geqq \sigma_{\infty}\right)+E_{x}\left(S_{t}^{2} ; t<\sigma_{\infty}\right)+E_{x}\left(v\left(X_{t}\right) ; t<\sigma_{\infty}\right),
$$

which implies that $v$ is an excessive function.

Moreover if $\mathrm{D}_{n}, n=1,2, \ldots$, are open subsets of $\mathrm{D}$ such that $\overline{\mathrm{D}}_{n} \subset \mathrm{D}_{n+1}$, $\mathrm{D}_{n} \uparrow \mathrm{D}(n \uparrow+\infty)$ and if $\sigma_{n}$ is the first leaving time from $\mathrm{D}_{n}$ of the path, then

$$
E_{x}\left(v\left(X_{\sigma_{n}}\right)\right)=v(x)-E_{x}\left(S_{\sigma n}^{2}\right) \longrightarrow 0 \quad(n \rightarrow+\infty) .
$$

Thus $v$ is a potential, that is, there exists a non negative measure $\nu$ on $\mathrm{D}$ such that

$$
v(x)=\int_{\mathrm{D}} G(x, y)_{\nu}(d y) .
$$

Denote by $\mathbf{D}(u)$ the total mass of $\nu$ which is uniquely determined by $u^{3{ }^{3}}$

LEMma 3. It holds that

$$
\mathbf{D}(u)=\lim _{\alpha \rightarrow+\infty} \alpha \int_{\partial \mathrm{D}} \int_{\mathrm{D}} h_{\alpha}(\xi, x) v(x) d x d \xi
$$

Proof. By (10) and (12), we can see that

$$
\begin{aligned}
\alpha \int_{\partial \mathrm{D}} \int_{\mathrm{D}} h_{\alpha}(\xi, x) v(x) d x d \xi & =\int_{\partial \mathrm{D}} \int_{\mathrm{D}}\left(h(\xi, x)-h_{\alpha}(\xi, x)\right)_{\nu}(d x) d \xi \\
& \uparrow \int_{\partial \mathrm{D}} \int_{\mathrm{D}} h(\xi, x)_{\nu}(d x) d \xi=\nu(\mathrm{D}) \quad(\alpha \uparrow+\infty)
\end{aligned}
$$

3) $\nu(d x)=a^{i j}(x) \frac{\partial H u(x)}{\partial x^{i}} \frac{\partial H u(x)}{\partial x^{j}} d x$ at least when $u$ is the function of class $\mathbf{C}^{3}$. 
THEOREM 2. If $H u$ is the function of class $\mathbf{C}^{1}$ on, $\overline{\mathrm{D}}$, then

$$
\mathrm{D}(u)=\int_{\partial \mathrm{D}} \int_{\partial \mathrm{D}}(u(\xi)-u(\eta))^{2} U(\xi, \eta) d \xi d \eta \text {. }
$$

Proof. We find by simple caluculations,

$$
\begin{aligned}
& \int_{\partial \mathrm{D}} \int_{\partial \mathrm{D}}(u(\xi)-u(\eta))^{2} U_{\alpha}(\xi, \eta) d \xi d \eta-\alpha \int_{\partial \mathrm{D}} \int_{\mathrm{D}} h_{\alpha}(\xi, x) v(x) d x d \xi \\
= & \alpha \int_{\partial \mathrm{D}} \int_{\mathrm{D}}(H u(x)-u(\xi))^{2} h_{\alpha}(\xi, x) d x d \xi,
\end{aligned}
$$

for the continuous function $u$ on $\partial \mathrm{D}$.

According to Lemma 3, (14) is true if and only if the right side of (15) tends to zero as $\alpha$ tends to infinity.

Take a fixed Euclidean coodinate, by which we represent $x, \xi$ as $\left(x^{1}, x^{2}\right.$; $\left.\cdots x^{N}\right),\left(\xi^{1}, \xi^{2}, \ldots, \xi^{N}\right)$ respectively. When $H u$ is in the class $\mathbf{C}^{1}$ on $\bar{D}$, there exists a positive constant $C$ such that

$$
|H u(x)-u(\xi)| \leqq C \sum_{i=1}^{N}\left|x^{i}-\xi^{i}\right|, \quad \text { for any } x \in \mathrm{D} \text { and any } \xi \in \partial \mathrm{D} .
$$

Further relying upon the following estimate of the fundamental solution given in $[4]$,

$$
\frac{\partial}{\partial n_{\xi}} p(t, \xi, x) \leq\left(C_{1} t^{-1 / 2}+C_{2}\right) t^{-N / 2} \exp \left(-\frac{C_{3} \sum_{i=1}^{N}\left|x^{i}-\xi^{i}\right|^{2}}{4 t}\right), \quad x \in \mathrm{D}, \xi \in \partial \mathrm{D},
$$

$\left(C_{1}, C_{2}, C_{3}\right.$ are some positive constants), we can see for each $j,(1 \leqq j \leqq N)$,

$$
\begin{aligned}
& \alpha \int_{\mathrm{D}} h_{\alpha}(\xi, x)\left|x^{j}-\xi^{j}\right|^{2} d x^{1} d x^{2} \cdots d x^{N} \\
= & \alpha \int_{0}^{+\infty} e^{-\alpha t}\left[\int_{\mathrm{D}} \frac{\partial}{\partial n_{\bar{\xi}}} p(t, \xi, x)\left|x^{j}-\xi^{j}\right|^{2} d x^{1} d x^{2} \cdots d x^{N}\right] d t \leqq \frac{C^{\prime}}{\sqrt{\alpha}}, \quad(\alpha>1),
\end{aligned}
$$

where $C^{\prime}$ is a positive constant independent of $\xi$ and $\alpha$.

Therefore the right side of (15) vanishes as $\alpha$ tends to infinity.

\section{RefEREnCES}

[1] J. L. Doob, Conditional Brownian motion and the boundary limits of harmonic functions. Bull. Soc. Math. France, 85 (1957), 431-458.

[2] J. L. Doob, Boundary properties of functions with finite Dirichlet integrals. Annales 
Inst. Fourier, 12 (1962), 573-621.

[3] W. Feller, On boundaries and lateral conditions for the Kolmogorov differential equations. Ann. of Math., 65 (1957), 527-570.

[4] N. Ikeda, K. Sato, H. Tanaka and T. Ueno, Boundary problems of multidimensional diffusion processes, 1 . Seminar on probability, 5 (1960), (Japanese).

[5] S. Ito, Fundamental solutions of parabolic differential equations and boundary value problems. Jap. J. Math, 27 (1957), 55-102.

[6] H. Kunita and T. Watanabe, Markov chains and Martin boundaries. Sugaku, 142 (1962), 81-94 (Japanese).

[7] L. Nä̈m, Sur le rôle de la frontiere de R. S. Martin dans la théorie du potentiel. Annales Inst. Fourier, 7 (1957), 183-281.

[8] J. Neveu. Une generalisation des processus á accroissements positifs independents. Abh. Math. Sem. Univ. Hamburg, (1961), 36-61. 